\title{
Effects of Resistance Training on Ventricular Function and Hypertrophy in a Rat Model
}

\author{
Valério Garrone Barauna, MSc; Kaleizu Teodoro Rosa, MSc; \\ Maria Cláudia Irigoyen, PhD; and Edilamar Menezes de Oliveira, PhD
}

\begin{abstract}
Objective: The purpose of this study was to follow the ventricular function and cardiac hypertrophy in rats undergoing a resistance-training program for a period of 3 months.

Design: Forty animals were divided into two major groups: control $(n=16)$ and resistance trained $(n=24)$. From the resistance-trained group, 12 animals were resistance trained for 1 month and another 12 for 3 months. The resistance-training protocol was performed with 4 sets of 12 repetitions using $65 \%$ to $75 \%$ of one repetition maximum (maximum lifted weight with the exercise apparatus).
\end{abstract}

Methods: Echocardiographic analysis was performed at the beginning of the resistance-training period and at the end of each month. The repetition maximum was measured every 2 weeks. Cardiac hypertrophy was determined by echocardiography, by the absolute weight of the cardiac chambers and by histology of the left ventricle.

Results: Before resistance training, both groups had similar repetition maximums, ranging from I.8-fold to 2-fold the body weight; however, at the end of the resistance-training period, the repetition maximum of the resistance-trained group was 6 -fold greater than the body weight. The left ventricular mass as assessed by echocardiography was $8 \%, 12 \%$ and 16\% larger in the resistance-trained group than in the control group in the first, second and third months, respectively. This hypertrophy showed a similar increase in the interventricular septum and in the free posterior wall mass. There was no reduction in the end-diastolic left ventricular internal diameter during the 3-month resistance-training period. Systolic function did not differ between the groups throughout the resistance-training period.

Conclusion: Resistance training induces the development of concentric cardiac hypertrophy without ventricular dysfunction or cavity reduction. Although diastolic function was not completely investigated, we cannot exclude the possibility that resistance training results in diastolic dysfunction.

Keywords: Cardiac hypertrophy; Echocardiography; Resistance training

$\mathrm{R}$ raining is a specialize, also known as weight or strength increase muscle strength, muscle endurance and muscle power. In response to this kind of training, both skeletal and cardiac muscle adapts. The main muscle adaptation of the athlete's body is the increase in size or mass (hypertrophy) of type II skeletal muscle cells ${ }^{1}$ and cardiac hypertrophy. However, different from hypertensive conditions when pressure overload is continuous, the cardiovascular response

to this exercise model is characterized by the intermittent increase in blood pressure during exercise. ${ }^{2}$

Due to the pressure overload during exercise, practitioners of modalities such as weight training develop a cardiac hypertrophy different from those practitioners of sports with a high dynamic component (e.g., running). This hypertrophy is characterized by increases in the left ventricular (LV) wall and no changes in the diameter of the LV cavity in diastole. Thus, these athletes are presumed to develop concentric LV

Received: June 12, 2006

Revised: December 10, 2006

Accepted: January 18, 2007

doi: $10.3121 / \mathrm{cmr} .2007 .707$
Grant Support: Recipient of FAPESP Fellowship, $\mathrm{N}^{\circ} 03 / 10302-2$ (Barauna).

Supported by FAPESP grants 04/I 1624-6

(Oliveira) and 01/00009-0) (Irigoyen)

65, 05508-900, Butantã, Cidade Universitária, São Paulo, SP-Brazil

Tel: 55-||-309|-3|36, Fax: 55-II-38|3-592I,Email: edilamar@usp.br 
hypertrophy characterized by an increased ratio of wall thickness to radius. 3,4 The increase in wall thickness induced by pressure-overload is mainly due to an increase in the myocyte cross-sectional area. ${ }^{5}$

Cardiac hypertrophy might also result from pressure overload observed in many pathologic conditions such as hypertension. However, this kind of hypertrophy is followed by diastolic or systolic dysfunction and a disproportionate increase in the thickness of the LV posterior wall and interventricular septum. 6,7 Moreover, similar adaptations are usually found in athletes who use anabolic steroids associated with resistance training. 7,8

Although several authors ${ }^{3-5}$ have studied the impact of different sports on cardiac structure, a follow-up study using an animal model has not been used yet to study adaptive changes in resistance training. We chose to focus on the resistance-trained heart to investigate echocardiographic data, such as the association of LV mass with myocyte thickness and heart weight, and to investigate changes in ventricular function in rats undergoing resistance training for a period of 1 and 3 months.

\section{Methods}

Animal Care and Resistance-Training Protocol

Forty male Wistar rats (250-300 g, 10 weeks old) were randomly divided into two groups: control $(n=16)$ and resistance trained (trained) $(n=24)$. Half the animals were sacrificed after 1 month of training ( 8 controls and 12 trained) and the others after 3 months. Animals were housed in standard cages, and food and water were provided ad libitum. Animals were weighed every week. The environmental temperature was kept at $23 \pm 1{ }^{\circ} \mathrm{C}$, and a dark:light cycle of 12 hours each was maintained throughout the experiment. All protocols were in accordance with the guidelines of the Brazilian College for Animal Experimentation and were approved by the Biomedical Sciences Institute/USP-Ethical Committee for Animal Research.

Animals were exercised following a model described by Tamaki et al. ${ }^{9}$ Rats fitted with a canvas jacket were able to regulate the twisting and flexing of their torsos and were suspended in a standard position on their hind limbs. An electrical stimulation ( $20 \mathrm{~V}, 0.3 \mathrm{sec}$ duration at $3 \mathrm{sec}$ intervals) was applied to the rat's tail through a surface electrode. As a result, the rats flexed their legs repeatedly, which lifted the weight-arm of the training apparatus. Twelve animals from the trained group were exercised with 4 sets of 12 repetitions each with a $90 \mathrm{sec}$ rest period between each set, 5 times per week for 4 weeks, and the remaining animals in the group were exercised for 12 weeks. This exercise program started after 2 weeks of adaptation. After measurement of the maximum weight lifted (1 repetition maximum) with the squat-training apparatus, the training load was set at $65 \%$ to $75 \%$ of 1 repetition maximum. All training sessions were performed in a dark room. The maximum power generated for jumping in rats (1 repetition maximum) was defined as the maximum load that the rats were able to jump following electrical stimulation, and was measured biweekly.

\section{Echocardiography}

Echocardiographic features were obtained under the recommendations of the American Society of Echocardiography. ${ }^{10}$ Transthoracic echocardiography was performed before the training and every 30 days until the end of the third month, and by only one observer using Sequoia 512 equipment (ACUSON Corporation, Mountain View, CA) and a 10 to $14 \mathrm{MHz}$ multifrequency linear transducer. Images were obtained with the transducer placed on the animal's shaved chest (lateral recumbence). To optimize the image, a transmission gel was used between the transducer and the animal's chest (general imaging gel, ATL Inc., Reedsville, PA). Animals were scanned from below at a depth of $2 \mathrm{~cm}$ with the focus optimized at $1 \mathrm{~cm}$. All measurements were performed by the same observer based on the average of 3 consecutive cardiac cycles. Rats were anesthetized with halothane $(0.75 \%$ to $1.25 \%, 1 \mathrm{~L} / \mathrm{min})$. Wall thickness and $\mathrm{LV}$ dimensions were obtained from a short-axis view at the level of the papillary muscles. LV mass was calculated by the use of the following formula, assuming a spherical LV geometry and validated in rats: $\mathrm{LV}$ mass $=1,047 \mathrm{x}\left[(\mathrm{LVd}+\mathrm{PWd}+\mathrm{IWd})^{3}-\mathrm{LVd}^{3}\right]$, where 1,047 is the specific gravity of muscle, LVd is LV end-diastolic diameter and PWd and IWd are end-diastolic posterior and interseptum wall thickness, respectively. LV shortening was calculated as (LVd-LVs)/LVdx100, where LVs is LV end-systolic diameter. LV ejection fraction was calculated according to the Teichholz formula. Two-dimensionally guided pulsed Doppler recordings of LV transmitral flow were obtained from the apical 4-chamber view. Isovolumic relaxation time was taken as the time from aortic valve closure to the onset of mitral flow. Velocity of circumferential shortening was measured using the formula $(\mathrm{LVd}-\mathrm{LVs}) /(\mathrm{LvdxET})$, where ET is the ejection time.

\section{Morphological Analysis}

The hearts were excised and perfused through the aortic stump from a reservoir located $70 \mathrm{~cm}$ above the heart and kept at room temperature. All hearts were initially perfused with $10 \mathrm{ml}$ of phosphate buffered saline to wash out the blood, and after the hearts were stopped at diastole by perfusion with $14 \mathrm{mM} \mathrm{KCl}$. The left ventricle was obtained as that remaining after removal of both atria and the free wall of the right ventricle. Afterwards, the cardiac chamber was weighed.

For histological analysis, the left ventricle was fixed in $6 \%$ formaldehyde and embedded in paraffin, cut into $5 \mu \mathrm{m}$ sections at the level of the papillary muscle and subsequently stained with hematoxylin and eosin. Two randomly selected sections from each animal were visualized by light microscopy using an oil immersion objective with a calibrated magnification (400X). Myocytes with visible nuclei and intact cellular membranes were chosen for diameter determination. The width of individually isolated 
cardiomyocytes displayed on a viewing screen was manually traced across the middle of the nuclei with a digitizing pad and determined by a computer-assisted image analysis system (Quantimet 520, Cambridge Instruments, Cambridge, UK). For each animal, approximately 20 visual fields were analyzed.

\section{Statistical Analysis}

All data are expressed as mean \pm standard deviation (SD). Differences among groups (left ventricle, right ventricle and atrium weight) were assessed by the Student $t$ test. All other statistical comparisons were performed by a randomized repeated measure 1-way analysis of variance (ANOVA). When a significant difference was detected, comparisons were carried out by Tukey's post-hoc test. $P<0.05$ was considered statistically significant.

\section{Results}

No difference was observed between groups for body weight or heart rate during the 3 month study period (table 1 ).

\section{One Repetition Maximum}

Figure 1 shows a progressive increase in the absolute weight lifted by the trained group obtained during the repetition maximum test. Both the control and the trained group had similar values for repetition maximum at the beginning (day 0 ) of the protocol. After 15 days, the load lifted by the trained group was already higher than that of the control group and also $76 \%$ higher than that lifted in the first test. At the end of the first month, the trained group lifted $1488 \pm 83 \mathrm{~g}$, and 2350 $\pm 108 \mathrm{~g}$ at the end of the third month, which represents 4.7 -fold and 6.7 -fold the body weight for 1 and 3 months, respectively.

\section{Cardiac Function}

Echocardiographic results from cardiac function in control and trained animals are summarized in table 1 . Neither diastolic function analyzed by isovolumic relaxation time, nor systolic

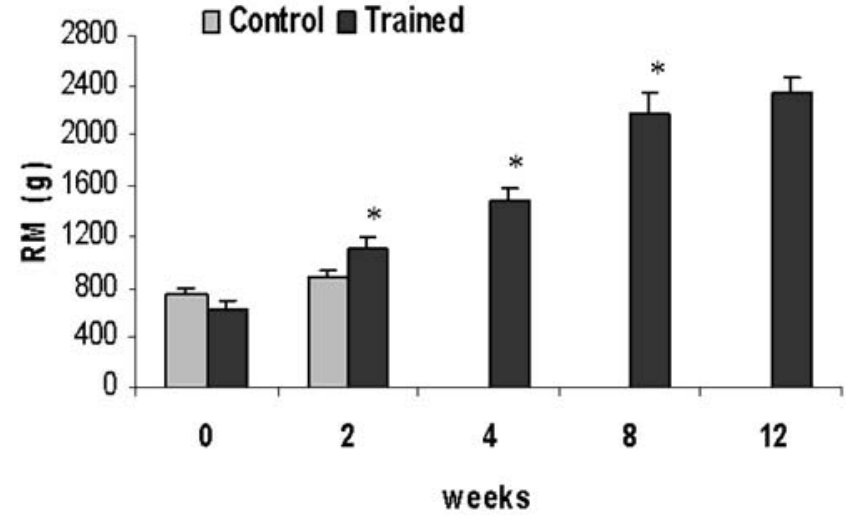

Figure 1. Absolute values for 1 repetition maximum test. Results are presented as mean \pm standard deviation. ${ }^{*} P<0.05$ when compared with the previous test. Randomized one-way ANOVA.

function analyzed by both LV ejection fraction and fractional shortening, and by velocity or circumferential fiber shortening were significantly different between the control and trained group during the 3 month training period.

\section{Cardiac Structure by Echocardiography}

The mean relative LV wall thickness of the control group was significantly smaller than that of the trained group in the second and third month. This may indicate the development of concentric hypertrophy. Results from the echocardiography studies are summarized in table 2 .

LV internal diameter, interventricular septum and LV posterior wall thickness were measured during the diastolic phase. No significant difference in LV internal diameter was observed when the control and trained groups were compared in each of the 3 months of the protocol. LV hypertrophy was similar in both walls $(1.4 \%, 7.1 \%$ and $8.3 \%$ in the first, second and third month, respectively). The difference between the control and

Table 1. Body weight, heart rate and cardiac function of control and trained rats during the 3-month training period. Results are presented as mean \pm SD.

\begin{tabular}{|c|c|c|c|c|c|c|c|c|}
\hline & \multicolumn{4}{|c|}{ Control $(n=8)$} & \multicolumn{4}{|c|}{ Trained $(n=12)$} \\
\hline & \multicolumn{4}{|c|}{ Month } & \multicolumn{4}{|c|}{ Month } \\
\hline & 0 & 1 & 2 & 3 & 0 & 1 & 2 & 3 \\
\hline BW, g & $306 \pm 24$ & $326 \pm 23$ & $368 \pm 28$ & $370 \pm 21$ & $302 \pm 19$ & $321 \pm 24$ & $347 \pm 23$ & $350 \pm 37$ \\
\hline $\mathrm{HR}, \mathrm{bpm}$ & $408 \pm 33$ & $420 \pm 15$ & $400 \pm 36$ & $423 \pm 21$ & $420 \pm 13$ & $410 \pm 17$ & $422 \pm 14$ & $417 \pm 19$ \\
\hline LVEF, \% & $54.8 \pm 1.9$ & $55.1 \pm 1.8$ & $53.7 \pm 1.6$ & $52.3 \pm 0.9$ & $52.6 \pm 1.8$ & $54.9 \pm 1.9$ & $54.5 \pm 1.9$ & $52.7 \pm 0.9$ \\
\hline LVFS, \% & $24.8 \pm 1.1$ & $24.9 \pm 1$ & $24.1 \pm 1$ & $23.3 \pm 0.4$ & $23.3 \pm 1.1$ & $24.8 \pm 1.1$ & $24.5 \pm 1.2$ & $23.5 \pm 0.5$ \\
\hline VCF, circ/sec & $19.7 \pm 1.7$ & $20.2 \pm 0.8$ & $20.8 \pm 1.7$ & $17.4 \pm 0.5$ & $18.5 \pm 0.6$ & $21.1 \pm 0.5$ & $17.5 \pm 1.8$ & $17.6 \pm 0.8$ \\
\hline IVRT, ms & $20.8 \pm 3.1$ & $21 \pm 1.2$ & $20.4 \pm 1.5$ & $21 \pm 1$ & $19.7 \pm 2.1$ & $20.3 \pm 1$ & $22.4 \pm 1.6$ & $22 \pm 1.6$ \\
\hline
\end{tabular}

BW, body weight; HR, heart rate; IVRT, isovolumetric relaxation time; LVEF, left ventricular ejection fraction; LVFS, left ventricular fractional shortening; VCF, velocity of circumferential fiber shortening. 
Table 2. Cardiac structure of the control and trained rats during the 3 month training period. Results are presented as mean \pm SD.

\begin{tabular}{|c|c|c|c|c|c|c|c|c|}
\hline & \multicolumn{4}{|c|}{ Control $(n=8)$} & \multicolumn{4}{|c|}{ Trained $(n=12)$} \\
\hline & \multicolumn{4}{|c|}{ Month } & \multicolumn{4}{|c|}{ Month } \\
\hline & 0 & 1 & 2 & 3 & 0 & 1 & 2 & 3 \\
\hline LVIDd, cm & $0.73 \pm 0.06$ & $0.72 \pm 0.05$ & $0.75 \pm 0.04$ & $0.77 \pm 0.03$ & $0.71 \pm 0.03$ & $0.72 \pm 0.04$ & $0.72 \pm 0.02$ & $0.75 \pm 0.03$ \\
\hline PWTd, cm & $0.138 \pm 0.008$ & $0.138 \pm 0.004$ & $0.140 \pm 0.002$ & $0.143 \pm 0.005$ & $0.138 \pm 0.005$ & $0.140 \pm 0.002$ & $0.155 \pm 0.005^{\star}$ & $0.155 \pm 0.004^{*}$ \\
\hline IVSTd, cm & $0.138 \pm 0.007$ & $0.138 \pm 0.004$ & $0.140 \pm 0.001$ & $0.143 \pm 0.006$ & $0.138 \pm 0.004$ & $0.140 \pm 0.002$ & $0.155 \pm 0.004^{*}$ & $0.155 \pm 0.005^{*}$ \\
\hline LVM, g & $0.59 \pm 0.04$ & $0.62 \pm 0.07$ & $0.68 \pm 0.06$ & $0.72 \pm 0.03$ & $0.61 \pm 0.02$ & $0.67 \pm 0.06$ & $0.76 \pm 0.02^{*}$ & $0.84 \pm 0.06^{\star}$ \\
\hline RWT & $0.38 \pm 0.023$ & $0.38 \pm 0.022$ & $0.37 \pm 0.022$ & $0.37 \pm 0.024$ & $0.39 \pm 0.021$ & $0.39 \pm 0.022$ & $0.43 \pm 0.024^{*}$ & $0.42 \pm 0.022^{*}$ \\
\hline
\end{tabular}

IVSTd, diastolic interventricular septum thickness; LVIDd, left ventricular end-diastolic internal diameter; LVM, left ventricular mass; PWTd, diastolic posterior wall thickness; RWT, relative wall thickness. ${ }^{*} P<0.05, \mathrm{TR} \times \mathrm{CO}$ group at the same age.

trained groups began in the second month and was maintained in the third month (table 2).

LV mass of the control rats was significantly smaller than the mean LV mass of the trained rats starting in the second month (11.7\% and $16.7 \%$ in the second and third month, respectively, both $P<0.05$ ) (table 2). However, in the first month, no significant difference was found; the LV mass only showed a slight increase $(8 \%)$.

Cardiac Morphology by Absolute Weight and Histology

A similar percentage of cardiac hypertrophy was found in the absolute LV weight (figure 2) $(7.6 \%$ in the first month, $P>0.05$; and $13.9 \%, P<0.05$ in the third month). No difference was found in the right ventricle or in the atrium weights. The increase in LV weight was further confirmed by increases of $8.3 \%$ and $14.1 \%$ in the LV myocyte width in the first and the third month, respectively, when control rats were compared with trained rats (figure 3).

\section{Discussion}

This is the first follow-up study using echocardiography in rats undergoing resistance training. The LV hypertrophy was

\section{$\square C O 1 \square T R 1 \square C O 3 \square T R 3$}

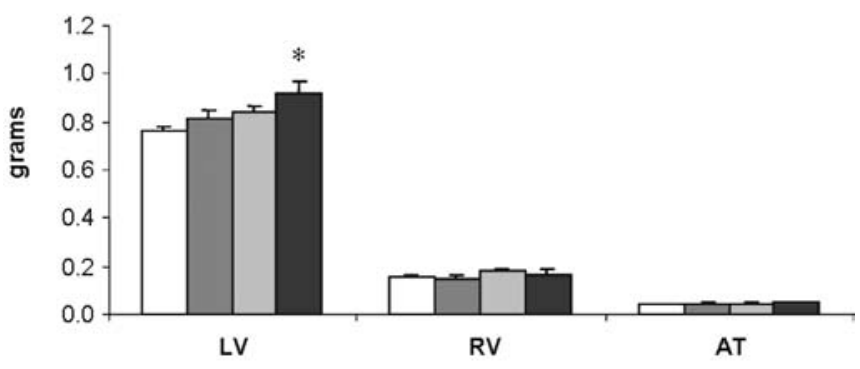

Figure 2. Absolute cardiac chamber weights. LV indicates left ventricle; $\mathrm{RV}$, right ventricle; $\mathrm{AT}$, atrium; $\mathrm{CO} 1$ and $\mathrm{CO} 3$, 1-month and 3-month control groups; TR 1 and TR 3, 1-month and 3-month trained groups. * $P<0.05$, TR $1 \times$ CO 1 and TR 3 x CO 3. Randomized Student $t$-test. similar when evaluated by echocardiography or absolute weight and cardiomyocyte width. This study showed a similar increase in the septum and free posterior wall mass but no reduction in the end-diastolic LV internal diameter during the 3 month training period, thus resulting in an increase in relative LV wall thickness. Also, diastolic function assessed by isovolumetric relaxation time, and systolic function assessed by ejection fraction, shortening fraction and velocity of circumferential fiber shortening did not differ between control and trained groups during the training period. These results suggest that resistance training induces concentric cardiac hypertrophy without any trace of ventricular dysfunction or reduction in the size of the ventricular cavity. Furthermore, echocardiography is a well-founded approach to evaluate cardiovascular adaptations in an experimental model that mimics squatting exercises performed by humans.

\section{Repetition Maximum}

To characterize training efficiency, repetition maximum was used as an index. Additionally, the maximum strength achieved by the rats was used to determine the training load and to demonstrate training development. As in aerobic training, where maximum heart rate or $\mathrm{VO}_{2 \max }$ are used to prescribe

\section{$\square$ CO $1 \square$ TR $1 \square C O 3 \square$ TR 3}

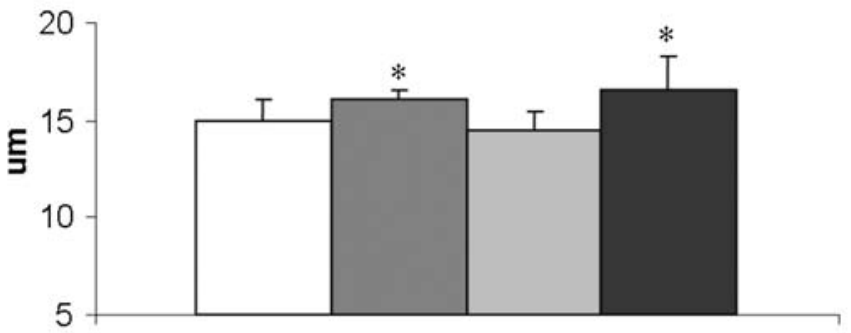

Figure 3. Left ventricular myocyte width. $\mathrm{CO} 1$ and $\mathrm{CO} 3$, 1-month and 3-month control groups; TR 1 and TR 3, 1-month and 3-month trained groups. ${ }^{*} P<0.05$, TR $1 \times$ CO 1 and TR 3 x CO 3. Randomized Student $t$-test. 
exercise ${ }^{11}$ repetition maximum is used in resistance training for prescription. ${ }^{12}$ Also, as previously demonstrated, results from our laboratory showed that this protocol is unaffected by the electrical stimulation. ${ }^{13}$

The results shown here are similar to results from other studies that used the same training apparatus. ${ }^{14}$ The increase in strength may have two origins, neural and muscular, as proposed by Moritani and deVries. ${ }^{15}$ They proposed that neural adaptations dominate early in the training program. Later, as neural adaptations reach a plateau, muscular adaptations (hypertrophy) dominate.

\section{Cardiac Structure and LV Mass}

Concerning cardiac structure of the athlete's heart, two different morphological forms may be distinguished. In sports with high dynamic and low static demand, eccentric hypertrophy predominates, while in strength training concentric hypertrophy is predominant. ${ }^{3}$ Fagard ${ }^{16,17}$ observed that athletes engaged in static training had LV dimension increased $2.5 \%$ and $\mathrm{LV}$ wall thickness increased $12 \%$ compared with that in non-athletes. In addition, Dickerman et al $^{18}$ demonstrated that LV wall thickness $>13 \mathrm{~mm}$ can be found routinely in elite resistance-trained athletes, and the use of anabolic steroids concomitant with resistance training does appear to augment LV mass. Normal or a slight increase in LV internal diameter and a large increase in LV wall thickness are commonly explained on the basis of the acute increase in blood pressure during resistance training. ${ }^{19,20}$

Also resistance-trained individuals have both the posterior LV wall thickness and intraventricular septum wall thickness greater than average. 17,21 However, these values do not exceed the limits of normal values, different from those observed in pathologic conditions, such as aortic stenosis, obstructive cardiomyopathy and hypertension.7,20 Therefore, the increase in the wall thickness is not a required result of all resistance training programs, and the conflicting results in the literature are probably due to differences in sex, age or in the training protocols used, including muscle mass involved, exercise intensity, rest periods between sets and training volume. ${ }^{22}$ Haykowsky et $\mathrm{al}^{21}$ have recently attributed this disparity between studies of whether resistance training consistently results in cardiac hypertrophy to three reasons: (1) acute cardiopulmonary mechanisms that minimize the increase in transmural pressure and LV wall stress during exercise, (2) the underlying use of anabolic steroids by the athletes, or (3) the specific type of resistance training performed. We believe that this exercise model may be a useful tool to unravel this discrepancy in an animal model, where the use of anabolic steroids and the type of resistance training may be precisely controlled and the cardiac ultra-structure further investigated.

Changes in the LV internal diameter in most cases are not significantly different from normal. 3,4,7,16,23,24 Although resistance training may have some effect on $\mathrm{LV}$ internal diameter, it is not reduced due to the pressure overload as in hypertension or other forms of induced cardiomyopathy pressure-overload. ${ }^{7}$

As observed above, LV mass can increase due to an increase in either ventricular wall thickness or internal dimensions. The latter is frequently observed when the primary stimulus is the volume overload. Increased end-diastolic wall stress leads to series replication of sarcomeres, fiber elongations, chamber enlargement and eccentric hypertrophy. Acute increase in peak systolic wall stress (pressure overload) leads to parallel replication of sarcomeres, wall thickening and concentric hypertrophy. $5,25,26$ Both cross-sectional studies and short-term longitudinal studies $3,17,21$ indicate that the absolute LV mass increases significantly due to resistance training. The similarities in the results among the three different methods of analysis of LV hypertrophy demonstrate that echocardiography is a reliable method to evaluate it also in the animal model.

\section{Cardiac Function}

LV systolic function is generally assessed in echocardiographic studies by measuring the extent of fiber shortening, ejection fraction and velocity of circumferential fiber shortening, ${ }^{27}$ while diastolic function is assessed by studying the pattern of ventricular filling through the mitral valve. ${ }^{28}$ Abnormalities in systolic and diastolic function are generally associated with cardiac hypertrophy induced by pathological conditions, such as hypertension and valvular disease.7,29 Our results of systolic function are in agreement with those in the literature where reports demonstrate that cardiac function is not altered in resistance-trained individuals. ${ }^{3,4,7}$ However, to the best of our knowledge, only two studies indicate an enhanced systolic function. ${ }^{30,31}$ One study indicated enhanced diastolic function, reporting that atrial peak-filling rate is greater in power lifters than in non-power lifters. ${ }^{32}$

\section{Clinical Implications of the Study}

Physical activity can potentially be an effective treatment for various clinical conditions, such as cardiovascular disease and osteoarthritis. In recent years, an increasing number of clinical and research experiments have been conducted on resistance exercise in congestive heart failure. Patients with congestive heart failure have a poor clinical status and impaired exercise capacity due to both cardiac limitations and peripheral maladaptations of the skeletal musculature. Levinger et $\mathrm{al}^{33}$ investigated the effects of 8 weeks of resistance training on the $\mathrm{LV}$ structure and function of patients with congestive heart failure. They reported that resistance training is a suitable method of exercise for congestive heart failure patients since it does not cause a reduction of LV contractility function or enhance myocardial deterioration as measured by ejection fraction and fractional shortening.

Recent studies ${ }^{34,35}$ indicate that endothelial dysfunction in coronary and also peripheral arteries predicts cardiovascular 
events; however, the mechanisms responsible for the benefits of resistance training on endothelial function are controversial. It is not clear, however, whether the beneficial effects of resistance training on endothelial function are a consequence of improvements in risk factors (serum lipid concentration, blood pressure, obesity or glycemic control) induced by resistance training or to some other mechanisms. However, Green et $\mathrm{al}^{36}$ show that the benefits of resistance training on vascular function are not solely mediated by the effects of exercise on cardiovascular risk factors. An explanation for the effect of exercise training on vasculature function is that exercise exerts direct effects on the vasculature by increasing shear stress that increases vascular nitric oxide production. Vascular function might be considered a useful independent measure of atherosclerotic disease risk. ${ }^{34,35}$ According to recent studies, combined endurance and resistance training or resistance training alone should be recommended as a safe and effective alternative training mode in order to counteract peripheral maladaptation and improve muscle strength necessary for daily activities, and thus improve the quality of life of congestive heart failure patients.

\section{Study Limitations}

Although it is well established that the anesthetic regimen modifies cardiac parameters, such as heart rate and ejection fraction, performing an echocardiogram in awake animals is challenging. We chose to do the echocardiographic examination by using halothane anesthesia in an attempt to keep the heart rate as close as possible to the physiologic state. However, high heart rates, common in rats, are used to unite $\mathrm{E}$ and A peaks and make isovolumic relaxation time too short, which makes diastolic function measurements unavailable. However, having all animals undergo the same anesthetic regimen makes the differences observed trustworthy. Also, as we have followed the animals for only 3 months, we cannot exclude changes occurring over a long period.

\section{Conclusion}

The main findings in this study indicate that resistance training in rats, which mimics squatting exercises performed by humans, induces chronic adaptations, such as cardiac hypertrophy, in the first, second and third month of training. It also indicates that echocardiography is a well-founded approach to evaluate these cardiovascular adaptations in this experimental model.

In conclusion, our results suggest that resistance training induces concentric cardiac hypertrophy without systolic dysfunction or reduction in the ventricular cavity. The similarities between previous reports having humans as subjects and our findings indicate that the model may be used for further studies concerning the development of physiological and pathological concentric cardiac hypertrophy.

\section{Acknowledgments}

We are grateful to Dr. J.G. Mill for review of this manuscript.

\section{References}

1. Komi PV. Strength and power in sports. 2nd ed. New York, NY: Blackwell Science, 2003.

2. Effron MB. Effects of resistive training on left ventricular function. Med Sci Sports Exerc 1989;21:694-697.

3. Pluim BM, Zwinderman AH, van der Laarse A, van der Wall EE. The athlete's heart. A meta-analysis of cardiac structure and function. Circulation 2000;101:336-344.

4. Longhurst JC, Kelly AR, Gonyea WJ, Mitchell JH. Echocardiographic left ventricular masses in distance runners and weight lifters. J Appl Physiol 1980;48:154-162.

5. Grossman W, Jones D, McLaurin LP. Wall stress and patterns of hypertrophy in the human left ventricle. J Clin Invest 1975;56:56-64.

6. Shapiro LM, Gibson DG. Patterns of diastolic dysfunction in left ventricular hypertrophy. Br Heart J 1988;59:438-445.

7. Hildick-Smith DJ, Shapiro LM. Echocardiographic differentiation of pathological and physiological left ventricular hypertrophy. Heart 2001;85:615-619.

8. Grace F, Sculthorpe N, Baker J, Davies B. Blood pressure and rate pressure product response in males using high-dose anabolic androgenic steroids (AAS). J Sci Med Sport 2003;6:307-312.

9. Tamaki T, Uchiyama S, Nakano S. A weight-lifting exercise model for inducing hypertrophy in the hindlimb muscles of rats. Med Sci Sports Exerc 1992;24:881-886.

10. Sahn DJ, DeMaria A, Kisslo J, Weyman A. Recommendations regarding quantitation in M-mode echocardiography: results of a survey of echocardiographic measurements. Circulation 1978;58:1072-1083.

11. Zavorsky GS. Evidence and possible mechanisms of altered maximum heart rate with endurance training and tapering. Sports Med 2000;29:13-26.

12. Fernandez R. One repetition maximum clarified. J Orthop Sports Phys Ther 2001;31:264.

13. Barauna VG, Junior ML, Costa Rosa LF, Casarini DE, Krieger JE, Oliveira EM. Cardiovascular adaptations in rats submitted to a resistance-training model. Clin Exp Pharmacol Physiol 2005;32:249-254.

14. Yaspelkis BB 3rd, Singh MK, Trevino B, Krisan AD, Collins DE. Resistance training increases glucose uptake and transport in rat skeletal muscle. Acta Physiol Scand 2002;175:315-323.

15. Moritani T, deVries HA. Neural factors versus hypertrophy in the time course of muscle strength gain. Am J Phys Med 1979;58:115-130.

16. Fagard RH. Athlete's heart: a meta-analysis of the echocardiographic experience. Int J Sports Med 1996;17:S140-S144.

17. Fagard RH. Impact of different sports and training on cardiac structure and function. Cardiol Clin 1997;15:397-412.

18. Dickerman RD, Schaller F, McConathy WJ. Left ventricular wall thickening does occur in elite power athletes with or without anabolic steroid use. Cardiology 1998;90:145-148.

19. Fleck SJ. Cardiovascular adaptations to resistance training. Med Sci Sports Exerc 1988;20:S146-S151.

20. MacDougall JD, McKelvie RS, Moroz DE, Sale DG, McCartney N, Buick F. Factors affecting blood pressure during heavy weight lifting and static contractions. J Appl Physiol 1992;73:1590-1597.

21. Haykowsky MJ, Quinney HA, Gillis R, Thompson CR. Left ventricular morphology in junior and master resistance trained athletes. Med Sci Sports Exerc 2000;32:349-352. 
22. Urhausen A, Kindermann W. Echocardiographic findings in strength- and endurance-trained athletes. Sports Med 1992;13:270-284.

23. Wolfe LA, Cunningham DA, Boughner DR. Physical conditioning effects on cardiac dimensions: a review of echocardiographic studies. Can J Appl Sport Sci 1986;11:66-79.

24. George KP, Wolfe LA, Burggraf GW, Norman R. Electrocardiographic and echocardiographic characteristics of female athletes. Med Sci Sports Exerc 1995;27:1362-1370.

25. Perrault H, Turcotte RA. Exercise-induced cardiac hypertrophy. Fact or fallacy? Sports Med 1994;17:288-308

26. Shapiro LM. Physiological left ventricular hypertrophy. Br Heart J 1984;52:130-135.

27. Spirito P, Pelliccia A, Proschan MA, Granata M, Spataro A, Bellone P, Caselli G, Biffi A, Vecchio C, Maron BJ. Morphology of the "athlete's heart" assessed by echocardiography in 947 elite athletes representing 27 sports. Am J Cardiol 1994;74:802-806.

28. Shimizu G, Hirota Y, Kita Y, Kawamura K, Saito T, Gaasch WH. Left ventricular midwall mechanics in systemic arterial hypertension. Myocardial function is depressed in pressure-overload hypertrophy. Circulation 1991;83:1676-1684.

29. Nishimura RA, Housmans PR, Hatle LK, Tajik AJ. Assessment of diastolic function of the heart: background and current applications of Doppler echocardiography. Part I. Physiologic and pathophysiologic features. Mayo Clin Proc 1989;64:71-81.

30. Colan SD, Sanders SP, Borow KM. Physiologic hypertrophy: effects on left ventricular systolic mechanics in athletes. J Am Coll Cardiol 1987;9:776-783.

31. Maron BJ. Hypertrophic cardiomyopathy: a systematic review. JAMA 2002;287:1308-1320.

32. MacDougall JD, Tuxen D, Sale DG, Moroz JR, Sutton JR. Arterial blood pressure response to heavy resistance exercise. J Appl Physiol 1985;58:785-790.

33. Levinger I, Bronks R, Cody DV, Linton I, Davie A. The effect of resistance training on left ventricular function and structure of patients with chronic heart failure. Int J Cardiol 2005; 105:159-163.

34. Suwaidi JA, Hamasaki S, Higano ST, Nishimura RA, Holmes DR Jr, Lerman A. Long-term follow-up of patients with mild coronary artery disease and endothelial dysfunction. Circulation 2000;101:948-954.

35. Vita JA, Keaney JF Jr. Endothelial function: a barometer for cardiovascular risk? Circulation 2002;106:640-642.

36. Green DJ, Walsh JH, Maiorana A, Best MJ, Taylor RR, O'Driscoll JG. Exercise-induced improvement in endothelial dysfunction is not mediated by changes in $\mathrm{CV}$ risk factors: pooled analysis of diverse patient populations. Am J Physiol Heart Circ Physiol 2003;285:H2679-H2687.

\section{Author Affiliations}

Valério Garrone Barauna, MSc

School of Physical Education and Sports

University of São Paulo

São Paulo, Brazil

Kaleizu Teodoro Rosa, MSc

Heart Institute (InCor)

University of São Paulo, Medical School

São Paulo, Brazil

Maria Cláudia Irigoyen, PhD

Heart Institute (InCor)

University of São Paulo, Medical School

São Paulo, Brazil

Edilamar Menezes de Oliveira, PhD

School of Physical Education and Sports

University of São Paulo

São Paulo, Brazil 